\title{
Intrastromal Corneal Ring Segments Implantation in Patients with Mild Keratoconus
}

\author{
${ }^{1}$ Pablo F Rodrigues, ${ }^{2}$ Guilherme Ferrara, ${ }^{3}$ Paulo Ferrara, ${ }^{4}$ Wagner G Dias, ${ }^{5}$ Leonardo Torquetti
}

\section{ABSTRACT}

Purpose: The purpose of this study is to evaluate the topographic, topometric and visual changes after implantation of Ferrara intrastromal corneal ring segments (ICRS) in grades I and II keratoconus patients.

Materials and methods: The chart records of 50 consecutively operated keratoconus patients were reviewed. The patients were operated on by the same surgeon, with the manual technique. All patients were preoperatively and postoperatively evaluated with the Pentacam (OCULUS Optikgeräte, Wetzlar, Germany). The studied parameters were: uncorrected distance visual acuity (UDVA), corrected distance visual acuity (CDVA), keratometry, corneal asphericity and corneal volume.

Results: Fifty eyes of 42 patients ( 26 males and 16 females) were analyzed. The mean preoperative UDVA was 0.91 ranging from LogMAR 0.10 to 1.30. The mean postoperative CDVA was 0.19 ranging from 0.00 to 0.54 LogMAR. The postoperative CDVA was equal or better than 0.18 in 37 cases $(74 \%)$. The mean $\mathrm{K} 1$ decreased from 45.80D $( \pm 2.52)$ preoperatively to $44.27 \mathrm{D}( \pm 2.10)$ postoperatively and the mean K2 value, from 49.06D $( \pm 2.09)$ to $46.22 \mathrm{D}( \pm 1.89)$. The mean asphericity increased from -0.71 preoperatively to -0.29 postoperatively. The average preoperative corneal volume was $56.89 \pm 3.11 \mathrm{~mm}^{3}$ while the average postoperative corneal volume was $57.64 \pm 3.05 \mathrm{~mm}^{3}$.

Conclusion: The study supports the early indication of implantation of Ferrara ICRS in mild to moderate keratoconus cases in order to achieve good visual, keratometric and ashpericity outcomes.

Keywords: Mild keratoconus, Intrastromal corneal ring segment, Corneal volume, Asphericity.

How to cite this article: Rodrigues PF, Ferrara G, Ferrara P, Dias WG, Torquetti L. Intrastromal Corneal Ring Segments Implantation in Patients with Mild Keratoconus. Int J Kerat Ect Cor Dis 2014;3(3):122-126.

${ }^{1,2,5}$ Cornea Subspecialty, ${ }^{3}$ Director, ${ }^{4}$ Professor

${ }^{1}$ Department of Ophthalmology and Visual Sciences, Federal University of São Paulo, SP, Brazil

${ }^{2,3}$ Department of Ophthalmology, Dr Paulo Ferrara Eye Clinic Belo Horizonte, MG, Brazil

${ }^{4}$ Sector of Cornea and Cataract Hilton Rocha Institute, Belo Horizonte, MG, Brazil

${ }^{5}$ Department of Ophthalmology, Center of Excellence in Ophthalmology, Pará de Minas, MG, Brazil

Corresponding Author: Pablo F Rodrigues, Department of Ophthalmology and Visual Sciences, 81 Benedito Lapin St Suit 101, Zip Code 04532-040, SP, Brazil, e-mail: drpablo. rodrigues@gmail.com

\section{Source of support: Nil}

Conflict of interest: None

\section{INTRODUCTION}

Keratoconus is a noninflammatory corneal condition in which there is thinning and protrusion of the cornea. It is commonly bilateral, involving two-third of the cornea, with its central apex just below the visual axis. The condition, along with pellucid marginal degeneration, keratoglobus and posterior keratoconus represents phenotypical variations of the same pathogenic mechanism. Asides from a significant variability in prevalence, it has an estimated incidence of 50 to 230 cases per 100,000. ${ }^{1}$ Initially, it is usually managed with the correction of ametropia through the use of glasses. In cases which glasses do not provide a satisfactory corrected visual acuity, rigid gas permeable contact lenses are used. They, in turn, create a new anterior refractive surface; however, they do not stop the progression of the disease. The use of intracorneal implants for high myopia correction was initially described at the beginning of the 60 seconds. ${ }^{1,2}$ Its application is an important tool for keratoconus patients who are intolerant to contact lenses. The Ferrara ring implant (1986) was first used in the correction of myopia of up to 15 diopters. Its applicability and reproducibility allowed for its use in keratoconus patients, irregular astigmatism and after corneal transplants. ${ }^{3-6}$

There is an important debate regarding the best moment in which intrastromal corneal ring segments (ICRS) should be implanted. Levinger et $\mathrm{al}^{7}$ showed significant reduction in myopia with improvement in regular astigmatism in initial and moderate keratoconus; Fahd et $\mathrm{al}^{8}$ also demonstrated good results in moderate and advanced keratoconus. Kahn et al demonstrated significant corneal flattening and improved patient contact lens tolerance after ICRS implants in advanced keratoconus. At this moment, there is no consensus in the current literature about the best moment to implant the ICRS in keratoconus patients.

The purpose of this study is to evaluate the topographic, topometric and visual changes after implantation of Ferrara (ICRS) in grades I and II keratoconus patients. 


\section{MATERIALS AND METHODS}

The chart records of 50 consecutively operated keratoconus patients were reviewed. The patients were operated on by the same surgeon (Paulo Ferrara) from February to November 2011. This study was approved by the Ethics Committee of the Instituto Suel Abujamra (Plataforma Brasil) [Suel Abujamra Institute (Brazil Platform)], and follows the tenets of the Declaration of Helsinki. All patients signed an inform consent allowing the procedure to be done.

Inclusion criteria utilized: Topographic diagnosis of grades I and II keratoconus (Amslwer-Krumeich), absence of any other previous ocular disease or surgery, absence of corneal opacity, pachymetry greater than 450 micra at the incision site and minimal age of 16 years.

All patients were preoperatively and postoperatively evaluated with the Pentacam (OCULUS Optikgeräte, Wetzlar, Germany) for evaluation of anterior segment parameters.

All patients were operated using the manual surgical technique as previously described. ${ }^{9}$

\section{INTRASTROMAL RING (ICRS) IMPLANTATION METHOD}

\section{Surgical Planning}

The selection of ICRS was done according to the Ferrara asphericity nomogram. ${ }^{9}$ The most commonly accepted value of normal corneal asphericity is about $-0.23 \pm 0.08 .^{10}$ In patients with unilateral keratoconus, the asphericity of the fellow eye should be used as the postoperative target. Thus, it will be calculated from the algebraic expression of the preoperative asphericity -0.23 . This will be the target postoperative asphericity.

\section{Surgical Technique}

All surgeries were performed according to the standard manual technique.

A reference point was marked in the center of cornea, while asking to the patient to look to a red light attached

Table 1: Correlation between the preoperative $Q$ and preoperative and postoperative CDVA (mean)

\begin{tabular}{|c|c|c|c|c|}
\hline$Q\left(30^{\circ}\right)$ Pre & $\begin{array}{l}\text { CDVA } \\
\text { Pre }\end{array}$ & Eyes & $\begin{array}{l}\text { CDVA } \\
\text { Post }\end{array}$ & $\begin{array}{l}\text { Q }\left(30^{\circ}\right) \\
\text { Post }\end{array}$ \\
\hline$Q$ up to -0.3 & 0.84 & 4 & 0.28 & 0.09 \\
\hline$-0.3 \leq Q<-0.5$ & 0.81 & 8 & 0.15 & -0.17 \\
\hline$-0.5 \leq Q<-0.7$ & 0.80 & 13 & 0.22 & -0.25 \\
\hline$-0.7 \leq Q<-0.9$ & 0.79 & 11 & 0.16 & -0.34 \\
\hline$-0.9 \leq Q<-1.1$ & 1.07 & 9 & 0.19 & -0.35 \\
\hline $\begin{array}{l}\text { Less than or } \\
\text { equal to }-1.1\end{array}$ & 1.18 & 5 & 0.23 & -0.77 \\
\hline Total & 0.91 & 50 & 0.19 & -0.29 \\
\hline
\end{tabular}

to surgical microscope (while turned off). The incision was made at the steepest meridian of the anterior cornea surface, using a calibrated diamond knife was set at approximately $80 \%$ of the mean corneal thickness determined by Pentacam. Corneal pockets were then created using the spreader hook. One semicircular dissector was placed sequentially into the lamellar pocket to be steadily advanced by a rotational movement (counterclockwise and clockwise dissectors). After completion of the tunnelization the ring was inserted inside the tunnels.

Postoperative evaluations were performed on the first day, 1 week, 1 and 3 months postoperatively.

\section{DATA ANALYSIS}

Evaluation of preoperative collected data and evaluation on the 1st and 3rd months of cornea analysis. The studied parameters were: corneal asphericity, volume, keratometry and visual acuity. The paired student t-test was used for statistical analysis.

\section{RESULTS}

Fifty eyes of 42 patients (26 males and 16 females) were analyzed. Of all of the surgeries performed 25 were on right eyes and 25 on left eyes. The results present preoperative and last follow-up data. The age of patients ranged from 13 to 63 years (the mean age was 30.40 years old \pm 9.15$)$. The mean preoperative corrected distance visual acuity (CDVA) was 0.91 (LogMAR) ranging from 0.10 to 1.30 . The mean postoperative CDVA was 0.19 ranging from 0.00 to 0.54 . The postoperative CDVA was equal or better than 0.18 in 37 cases (74\%).

An observation between preoperative and postoperative asphericity and CDVA was described (Table 1). Fortyeight percent of the eyes present asphericity between -0.5 and -0.9 and achieve a mean CDVA post 0.19 LogMAR.

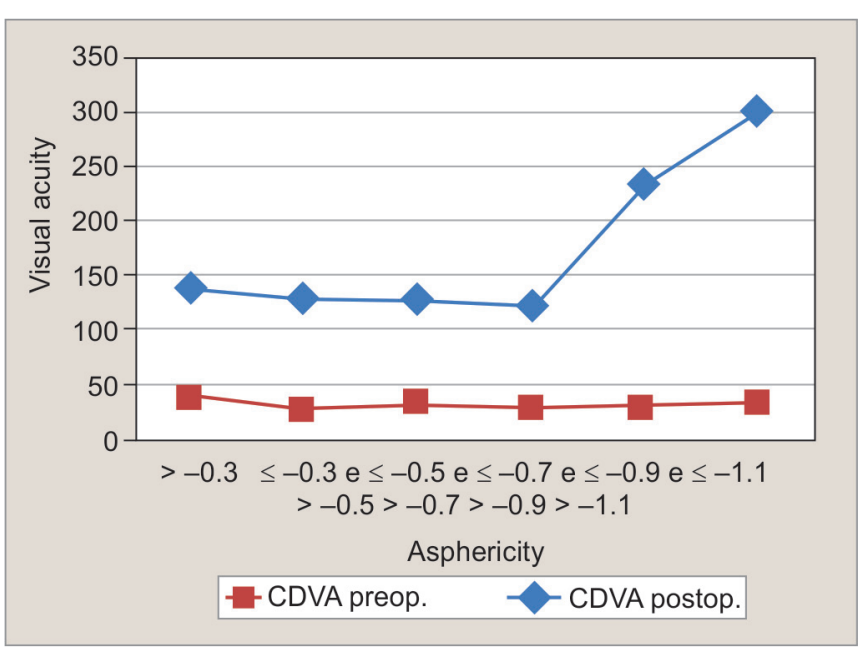

Graph 1: Correlation between corrected distance visual acuity and pre- and postoperative asphericity 
Table 2: $\mathrm{K}$ variation, $\mathrm{Q}$ variation and $\mathrm{CV}$ from preoperative to postoperative

\begin{tabular}{lllllll}
\hline & $K 1(D)$ & $K 2(D)$ & $K m(D)$ & $Q\left(30^{\circ}\right)$ & $C V$ & $p$-value \\
\hline Preop & $45.80 \pm 2.52$ & $49.06 \pm 2.09$ & $47.10 \pm 2.18$ & $-0.71 \pm 0.32$ & 56.89 & $<0.05$ \\
Postop & $44.27 \pm 2.10$ & $46.22 \pm 1.89$ & $45.18 \pm 1.88$ & $-0.29 \pm 0.29$ & $57.64 \pm 3.05$ & $<0.05$ \\
\hline
\end{tabular}

Table 3: Volume of each segment from thickness and its respective arc length

\begin{tabular}{lll}
\hline Volume & Thickness & Arc angle $\left(^{\circ}\right)$ \\
\hline 0.75 & 0.15 & 140 \\
0.94 & 0.20 & 140 \\
1.14 & 0.25 & 140 \\
0.85 & 0.15 & 160 \\
1.08 & 0.20 & 160 \\
1.30 & 0.25 & 160 \\
1.13 & 0.15 & 210 \\
1.42 & 0.20 & 210 \\
1.71 & 0.25 & 210 \\
\hline
\end{tabular}

There is great difficulty in measuring medical visual acuity (LogMAR chart) in patients with $\mathrm{Q}\left(30^{\circ}\right)$ equal to or less than 0.7 (Graph 1). The average CDVA in these patients was 1.0 still with a mean CDVA of 0.20. A CDVA better or equal to 0.18 occurred in $74 \%$ of all patients.

The mean K1 decreased from 45.80D $( \pm 2.52)$ preoperatively to $44.27 \mathrm{D}( \pm 2.10)$ postoperatively and the mean $\mathrm{K} 2$ value, from $49.06 \mathrm{D}( \pm 2.09)$ to $46.22 \mathrm{D}( \pm 1.89)$ ( $\mathrm{p}<0.05)$. The mean asphericity increased from -0.71 preoperatively to -0.29 postoperatively ( $\mathrm{p}<0.05$ ) (Table 2 ). Currently, the most commonly accepted value in a young adult population is approximately $-0.23 \pm 0.08$ measured at a $4.5 \mathrm{~mm}$ optical zone. ${ }^{10}$

Corneal volume $(\mathrm{CV})$ was recently identified as an additional screening factor for keratoconus. ${ }^{11-13}$ Significant differences in CV have been reported between normal and mode-rate keratoconic eyes (Pentacam system: $60.83 \pm 3.27 \mathrm{~mm}^{3}$ controls vs $57.98 \pm 2.65 \mathrm{~mm}^{3}$ moderate keratoconus). ${ }^{11}$

The average preoperative corneal volume was 56.89 $\pm 3.11 \mathrm{~mm}^{3}$ while the average postoperative corneal volume was $57.64 \pm 3.05 \mathrm{~mm}$. $^{3}$ For better analysis of this additional screening, we divided the eyes into three groups: group 1-patients with corneal volume lower than normal and group 2-patients with normal corneal volumes. The study included 19 cases, $38 \%$ of total cases, already showed a volume lower than normal before the procedures. Twenty-five eyes were in the normal corneal volume range before (ICRS implantation). The group 3 (comprised of 6 eyes) shows eyes that had an upper limit of normality before the ICRS.

We analyzed the volumetric behavior of the cornea after surgeries and obtained 22 eyes with normal corneal volume (vs 25 eyes before surgeries). This total included 21 eyes, which maintained of normal range corneal volume
Table 4: Volumetric comparison after discounting ICRS volume

\begin{tabular}{lll}
\hline & \multicolumn{2}{c}{ Volumetric comparison } \\
\cline { 2 - 3 } Keratometry & Reduction (\%) & Increase (\%) \\
\hline $\mathrm{Km} \geq 48 \mathrm{D}$ & 52 & 48 \\
$\mathrm{Km}<48 \mathrm{D}$ & 71 & 29 \\
Total & 60 & 40 \\
\hline
\end{tabular}

after the surgeries and originally belonged to group 2 (with normal range corneal volume), and one eye from group 1 (corneal volume lower than normal). Regarding the group 1, 11 cases increased the final volume being nine eyes were introduced in the normal corneal volume with normal asphericity in eight eyes of them (Table 3).

The group 1 showed a presurgical average keratometry of 46.45 vs of $42.33 \mathrm{D}$ after ICRS implantation with an average final asphericity of -0.29 (Qval preoperative -0.32 ). The group 2 reach an average keratometry $45.84 \mathrm{D}$ (vs a preoperative 47.89D) but the initial asphericity already were -0.76 and presented the final asphericity -0.32 . The group 3 showed a presurgical average keratometry of 46.22D vs an average keratometry of 44.53D after ICRS implantation with an average final asphericitiy of -0.21 (vs -0.67 preoperative).

The mean volume of the ICRS implants was $1.31 \mathrm{~mm}^{3} \pm$ $0.39 \mu \mathrm{m}$, with a variation between 0.75 to $1.71 \mathrm{~mm}^{3}$. The volumetric data of each ICRS segment were supplied by the company AJL (Miñano, Álava-Spain) manufacturer of the FerraraRings ${ }^{\circledR}$ orthoses (Table 3 ).

In the group 1 (19 eyes), 11 eyes presented a final volume larger than initial plus the ICRS implants being nine reached the normal range of volume, and eight presented final volume lower than initial plus the ICRS.

On the other hand, the group 2 (25 eyes) presented seven eyes a final volume bigger than initial plus the ICRS implants with asphericity of -0.34 but CDVA 0.15 vs 18 final volume lower than initial plus the ICRS with asphericity of -0.29 but CDVA 0.23 .

In the group 3 (6 eyes), two eyes presented a final volume bigger than initial plus the ICRS implants with asphericity of -0.08 but VA 0.09 vs four eyes wich final volume lower than initial plus the ICRS implants with asphericity of -0.27 but VA 0.18 .

An interesting relationship between volume and keratometry mean was observed from a $48 \mathrm{D}$. In $71 \%$ of patients that underwent surgery who presented a $\mathrm{Km}$ less or equal that $48 \mathrm{D}$ had a reduction in the final corneal volume (Table 4). 


\section{DISCUSSION}

Aydin et al demonstrated the low visual quality in keratoconus patients. ${ }^{14}$ This disease is progressive and vision may be seriously impaired, causing an important negative impact on patients' quality of life (QoL). ${ }^{15}$ A study conducted by the CLEK concluded that QoL is seriously affected in keratoconus patients and continues to decline over time, but an earlier study by our group showed that ICRS implantation improves visual QoL (V-QoL). ${ }^{16}$ The correlation between with quality of life (V-QoL) following ICRS implantation in these patients was established. ${ }^{17}$

The results demonstrated the low visual acuity present in patients suffering from keratoconus already in the initial grades $(0.90$ with a variability of \pm 0.74$)$. The patients evaluated in the study presented a significant improvement in visual acuity (from 1.30 to 0.18 LogMAR), and significant corneal flattening, with the mean keratometry decreasing from 47.14 to $45.18 \mathrm{D}$. We believe that all of this is corroborated by the reduction in optical aberration indices, and consequently improvement in visual quality. ${ }^{18}$

Some studies agree that the human cornea Q (asphericity) values range from -0.01 to $-0.80 .{ }^{19}$ However, in our studies, we observed that if we adopted such values as a reference we would have $56 \%$ of the patients within 'normality' with a mean visual acuity of 0.79 LogMAR before ICRS, i.e. completely outside of normality. The asphericity showed an important and direct relationship with the visual acuity. The Figure 1 demonstrates the progressive worsening of visual acuity preoperative and asphericity. This characteristic is more easily measured up to approximately 20/125. Beyond this point, we believe the quantification of visual acuity (LogMAR chart) is relatively compromised. After ICRS implantation, $28 \%$ of eyes turn to the normal range of asphericity, and among these eyes, $71.42 \%$ had a final visual acuity better than or equal 20/30.

Asphericity before and after surgery is an important parameter to measure, as it allowed us to assert the significant change and variability, in the initial cases of keratoconus as well as their improvement after the surgical procedure. This data supports the predictability and effectiveness of the nomogram based on asphericity also in mild keratoconus cases.

The correlation between keratometry and asphericity is evident with a strong correlation, ${ }^{20}$ however, no such corresponding correlation was shown with the corneal volume.

We measured the cornea volume after the procedure in all cases and, $58 \%$ of cases, the corneal volume turned to normal values and, in these cases, we obtained a final asphericity -0.28 with a mean visual acuity 0.15 being better or equal to 0.10 LogMAR in $41 \%$.

For the volumetric evaluation, we considered the volume of each ICRS in the cornea and therefore calculate the difference between the final (Vf) and initial volume (Vi) plus the ICRS volume (Vic). The result we call 'volume error' (Ver). If the result was positive after the surgery, the final volume is higher than initial. On the other hand, if 'the volume error' was negative, it means that the cornea was lower than initial.

We observed a final volume lower than the initial volume in $38 \%$ of all patients. Therefore, in $60 \%$ of cases we obtained a corneal remodeling whose final volume was lower than the initial volume, being the preoperative Km was 47.42 and 45.58 after the surgery and average final asphericity of -0.31 . That suggests that the ICRS promote indirectly a kind of reorganization by collagen lamellas of corneal stroma contributing for better values of keratometry, asphericity and visual acuity, which raises the possibility of corneal shrinkage after the procedure. Such a presumption is corroborated based on other studies that demonstrated the formation of collagen and other extracellular matrix components ${ }^{21}$ after ICRS implantation.

Such characteristics lead us to consider the possible role of the ICRS in relation to the reorganization of collagen fibers allowing these ectasic corneas to resemble their primary form and, in this way, improve their visual performance.

The Ferrara ICRS demonstrates its effectiveness in the treatment of initial and moderate keratoconus. This ability can be verified through the application of the proposed nomogram based on an analysis and observation of asphericity as a remodeling parameter and corresponding corneal visual function.

\section{CONCLUSION}

The study supports the early indication of Ferrara ICRS in mild to moderate keratoconus cases in order to achieve good visual, keratometric and asphericity outcomes.

\section{REFERENCES}

1. Rabinowitz YS,Nesburn AB,McDonnell PJ. Videokeratography of the fellow eye in unilateral keratoconus. Ophthalmology 1993; 100(2):181-186.

2. Barraquer JI. Modification of refraction by means of intracorneal inclusions. Int Ophthalmol Clin 1966;6(1):53-78.

3. Holland DR, Maeda N, Hannush SB, Riveroll LH, Green MT, Klyce SD, Wilson SE. Unilateral keratoconus. Incidence and quantitative topographic analysis. Ophthalmology 1997;104(9): 1409-1413.

4. Lee LR, Hirst LW, Readshaw G. Clinical detection of unilateral keratoconus. Australian and New Zealand J Ophthalmol 1995;23(2):129-133. 
5. Copeman PW. Eczema and keratoconus. Br Med J 1965; 2(5468):977-979.

6. Nose W, Neves RA, Burris TE, Schanzlin DJ, Belfort R Jr. Intrastromal corneal ring: 12-month sighted myopic eyes. J Refrac Surg 1996;12(1):20-28.

7. Levinger S, Pokroy R. Keratoconus managed with intacs: one-year results. Archives of Ophthalmology 2005;123(10): 1308-1314.

8. Fahd DC, Jabbur NS, Awwad ST. Intrastromal corneal ring segment SK for moderate to severe keratoconus: a case series. J Refrac Surg 2012;28(10):701-705.

9. Torquetti L, Berbel RF, Ferrara P. Long-term follow-up of intrastromal corneal ring segments in keratoconus. J Cataract Refrac Surg 2009;35(10):1768-1773.

10. Yebra-Pimentel E, Gonzalez-Jeijome JM, Cervino A, Giraldez MJ, Gonzalez-Perez J, Parafita MA. Corneal asphericity in a young adult population. Clinical implications. Archivos de la Sociedad Espanola de Oftalmologia 2004;79(8): 385-392.

11. Ambrosio R Jr, Alonso RS, Luz A, Coca Velarde LG. Cornealthickness spatial profile and corneal-volume distribution: tomographic indices to detect keratoconus. J Cataract Refrac Surg 2006;32(11):1851-1859.

12. Pinero DP, Alio JL, Aleson A, Escaf Vergara M, Miranda M. Corneal volume, pachymetry, and correlation of anterior and posterior corneal shape in subclinical and different stages of clinical keratoconus. J Cataract Refrac Surg 2010;36(5): 814-825.

13. Emre S, Doganay S, Yologlu S. Evaluation of anterior segment parameters in keratoconic eyes measured with the Pentacam system. J Cataract Refrac Surg 2007;33(10):1708-1712.
14. Kurna AS, Altun A, Gencaga T, Akkaya S, Sengor T. Vision related quality of life in patients with keratoconus. J Ophthalmol 2014;2014:694542.

15. Kymes SM, Walline JJ, Zadnik K, Sterling J, Gordon MO. Collaborative longitudinal evaluation of keratoconus study G: changes in the quality-of-life of people with keratoconus. Am J Ophthalmol 2008;145(4):611-617.

16. de Freitas Santos Paranhos J, Avila MP, Paranhos A Jr, Schor P. Evaluation of the impact of intracorneal ring segments implantation on the quality of life of patients with keratoconus using the National Eye Institute Refractive Error Quality of life (NEI-RQL) instrument. Br J Ophthalmol 2010;94(1):101-105.

17. Sahebjada S, Fenwick EK, Xie J, Snibson GR, Daniell MD, Baird PN. Impact of keratoconus in the better eye and the worse eye on vision-related quality of life. Investigative Ophthalmology and Visual Science 2014;55(1):412-416.

18. Ferrara G, Torquetti L, Ferrara P, Merayo-Lloves J. Intrastromal corneal ring segments: visual outcomes from a large case series. Clin Experimental Ophthalmol 2012;40(5):433-439.

19. Davis WR, Raasch TW, Mitchell GL, Mutti DO, Zadnik K. Corneal asphericity and apical curvature in children: a cross-sectional and longitudinal evaluation. Investigative Ophthalmol Visual Sci 2005;46(6):1899-1906.

20. Torquetti L. Corneal asphericity changes after implantation of intrastromal ring segments in keratoconus. J Emmtropia 2010;1(4):178-181.

21. Maguen E, Rabinowitz YS, Regev L, Saghizadeh M, Sasaki T, Ljubimov AV. Alterations of extracellular matrix components and proteinases in human corneal buttons with INTACS for post-laser in situ keratomileusis keratectasia and keratoconus. Cornea 2008;27(5):565-573. 